\title{
Aplicação do protocolo Ottawa por estudantes de medicina e residentes de ortopedia em entorses de tornozelo em um hospital de trauma*
}

\section{Application of the Ottawa Protocol by Medical Students and Orthopedic Residents on Ankle Sprains at a Trauma Hospital}

\author{
José Marcos Lavrador Filho ${ }^{1}$ Paulo Henrique Vogt ${ }^{10}$ Alexandre Roberto Roman Coelho ${ }^{2}$ \\ Daniel Kyubin $\mathrm{Cho}^{2}$ João Luiz Vieira da Silva ${ }^{2}$ Patrick Willian Padoani ${ }^{3 \odot}$ \\ ${ }^{1}$ Departamento de Ortopedia e Traumatologia, Hospital do \\ Trabalhador, Curitiba, PR, Brasil \\ ${ }^{2}$ Departamento de Ortopedia e Traumatologia, Universidade Federal \\ do Paraná, Curitiba, PR, Brasil \\ Endereço para correspondência Paulo Henrique Vogt, Departamento \\ de Ortopedia e Traumatologia, Hospital do Trabalhador, Avenida \\ Republica Argentina, 4406, Curitiba, PR, 81050-000, Brasil \\ (e-mail: paulovogt90@gmail.com).
}

${ }^{3}$ Departamento de Ortopedia e Traumatologia, Clínica de Fraturas e Ortopedia XV, Curitiba, PR, Brasil

Rev Bras Ortop 2020;55(5):620-624.

\begin{abstract}
Resumo
Objetivo Verificar a aplicabilidade e a concordância das regras do tornozelo de Ottawa aplicadas por estudantes de medicina e residentes de ortopedia em um serviço de trauma terciário, validando assim as regras de Ottawa para utilização em solo brasileiro.

Método Estudo prospectivo, realizado em um hospital terciário, incluindo todos os pacientes com trauma torcional agudo da articulação tibiotársica. Os pacientes realizaram radiografias de tornozelo e/ou pé, tendo sido aplicado o questionário com as regras do tornozelo de Ottawa por acadêmicos e, na sequência, por residentes. As radiografias foram avaliadas por ortopedistas plantonistas e especialista em pé e tornozelo, sendo a opinião do especialista considerada padrão-ouro da análise.

Resultados Foram avaliados 263 pacientes e, após aplicados os critérios de inclusão estabelecidos, restaram para a avaliação 226 casos. A sensibilidade para detectar lesões e o valor preditivo negativo (VPN) foi de $100 \%$. O teste mais sensível e com maior

Palavras-chave

- fratura por entorse

- tornozelo

- pé

- radiografia VPN para ambos, acadêmicos e residentes, foi a palpação do maléolo lateral. O estudo apresentou potencial de redução de $30 \%$ no total de exames solicitados.

Conclusão Os dados demostraram aplicabilidade e concordância entre acadêmicos e residentes, o que permite a validação do protocolo de Ottawa nos atendimentos de urgência e emergência no Brasil.
\end{abstract}

Trabalho desenvolvido no Serviço de Ortopedia e Traumatologia do Hospital do Trabalhador, Curitiba, PR, Brasil.

recebido

13 de Junho de 2019

aceito

29 de Novembro de 2019
DOI https://doi.org/

10.1055/s-0040-1702957. ISSN 0102-3616.
Copyright $\odot 2020$ by Sociedade Brasileira License terms de Ortopedia e Traumatologia. Published by Thieme Revinter Publicações Ltda, Rio de Janeiro, Brazil 
Abstract

\author{
Keywords \\ - sprain fracture \\ - ankle \\ - foot \\ - radiography
}

Objective To verify the applicability and agreement of the Ottawa ankle rules applied by medical students and orthopedic residents in a tertiary trauma service thus validating the Ottawa protocol for use on Brazilian soil.

Prospective This was a prospective study, conducted in a tertiary hospital, including all patients with acute torsial trauma of the tibiotarsal joint. The patients underwent ankle and/or foot radiographs, and the questionnaire with the Ottawa ankle rules was applied by academics and, subsequently, by residents. The radiographs were evaluated by on-call orthopedists and specialist in foot and ankle, and the expert opinion was considered the gold standard for analysis.

Results Two hundred and sixty-three patients were evaluated, and, after application of the established inclusion criteria, 226 cases remained for evaluation. The sensitivity to detect lesions and negative predictive value (NPV) was $100 \%$. The most sensitive test with higher NPV for both academics and residents was palpation of the lateral malleolus. The study presented potential for a reduction of $30 \%$ in the total number of tests requested. Conclusion The data showed applicability and agreement between academics and residents, which allows for the validation of the Ottawa protocol in emergency care in Brazil.

\section{Introdução}

Os traumatismos do tornozelo apresentam-se como uma das queixas mais comuns dos pronto-atendimentos, correspondendo de 6 a $12 \%$ dos atendimentos ${ }^{1}$ e podendo acometer pacientes de diversas idades e ocorrer em traumas de baixa ou alta energia. Eles acometem aproximadamente 1/10.000 habitantes por dia no mundo ${ }^{2}$ e decorrem de movimentos cotidianos ou práticas esportivas, cursando com fraturas em $15 \%$ dos casos. $^{3}$

Observa-se como prática corrente nos serviços de urgência e emergência a solicitação de radiografias em incidências antero-posterior e perfil para a avaliação das lesões decorrentes do trauma torcional do tornozelo, apesar da literatura evidenciar que $85 \%$ são entorses sem lesão óssea. ${ }^{3}$

Buscando padronizar os critérios e reduzir as solicitações de radiografias, Stiell et al. ${ }^{3}$ desenvolveram, na década de 90 , as regras do tornozelo de Ottawa, constituído de uma análise clinica com critérios objetivos que indicam a necessidade de realizar radiografias nos traumas de tornozelo.

Há necessidade de radiografias do tornozelo no paciente que apresenta história de trauma torcional com dor maleolar referida associada a um ou mais dos seguintes critérios:

1. Dor maleolar lateral à palpação óssea $(6 \mathrm{~cm}$ distais na região posterior).

2. Dor maleolar medial à palpação óssea $(6 \mathrm{~cm}$ distais na região posterior).

3. Incapacidade de apoiar o membro (imediatamente após trauma e por 4 passos na urgência).

Da mesma maneira, recomenda-se a solicitação de radiografias do pé quando o paciente apresentar história de trauma torcional com dor referida no médio pé associada a um ou mais dos seguintes fatores:

1. Dor a palpação óssea da base do $5^{\circ}$ metatarso.

2. Dor a palpação óssea do navicular.
3. Incapacidade de apoiar o membro (imediatamente após trauma e por 4 passos na urgência).

É importante salientar que este protocolo não deve ser utilizado em pacientes com quadro de intoxicação aguda ou não cooperativos, politraumatizados, com diminuição da sensibilidade nos membros inferiores, com grande edema que dificulte a palpação dos maléolos, e menores de 18 anos. Os pacientes submetido ao protocolo e que optaram pela não realização de radiografias devem ser devidamente orientados, com informações por escrito em relação ao seguimento e necessidade de reavaliação caso persistam com sintomas.

No presente estudo, temos como objetivo verificar a aplicabilidade e a concordância das regras do tornozelo de Ottawa aplicadas por estudantes de medicina, em estágio supervisionado, e residentes de ortopedia após treinamento prévio em um serviço terciário de atendimento ao trauma na nossa cidade, validando assim as regras do tornozelo de Ottawa para utilização em solo brasileiro.

\section{Materiais e Métodos}

Trata-se de um estudo prospectivo, realizado no setor de urgências e emergências de um hospital terciário, com coleta de dados de setembro a novembro de 2014, incluindo todos os pacientes com trauma torcional da articulação tibiotársica com menos de 24 horas de evolução.

O projeto foi aprovado e supervisionado pelo comitê de ética da instituição na qual ocorreu a aplicação do protocolo e a coleta de dados.

Os critérios de exclusão para análise estatística foram os casos de radiografias inadequadas ou que apresentavam fise aberta. Foram excluídos da coleta dos dados casos politraumatizados ou vítimas de traumatismos de alta energia.

Todos os pacientes realizaram radiografias de tornozelo e/ou pé nas incidências anteroposterior e perfil, tendo sido aplicado o questionário com as regras do tornozelo de 
Tabela 1 Características da amostra $(n=263)$

\begin{tabular}{|l|l|l|l|}
\hline Variável & Categoria & $\mathbf{n}$ & $\%$ \\
\hline \multirow{3}{*}{ Sexo } & Masculino & 133 & 50,6 \\
\cline { 2 - 4 } & Feminino & 130 & 49,4 \\
\hline \multirow{3}{*}{ Faixa etária } & $\leq 54$ anos & 240 & 91,0 \\
\cline { 2 - 4 } & $\geq 55$ anos & 23 & 9,0 \\
\hline \multirow{3}{*}{ Fise aberta } & Não & 226 & 86,3 \\
\cline { 2 - 4 } & Sim & 37 & 13,7 \\
\hline \multirow{3}{*}{\begin{tabular}{l} 
entrário de \\
\cline { 2 - 4 }
\end{tabular}} & & 94 & 35,7 \\
\cline { 2 - 4 } & Manhã & 105 & 39,9 \\
\cline { 2 - 4 } & Tarde & 64 & 24,4 \\
\cline { 2 - 4 } & Noite & & \\
\hline
\end{tabular}

Ottawa por acadêmicos de medicina inscritos no estágio voluntário de traumatologia do hospital e, na sequência, por residentes do serviço de ortopedia e traumatologia.

As radiografias foram avaliadas por ortopedistas plantonistas, os quais conduziram o tratamento dos casos e, posteriormente, por um ortopedista especialista em pé e tornozelo, e a resposta foi dicotomizada em não ou sim para avaliação estatística, sendo a opinião do especialista considerada padrão-ouro da análise.

Foram utilizadas as variáves de sexo, faixa etária $(\leq 54$ anos e $\geq 55$ anos), presença de fise óssea aberta (não ou sim), e período do atendimento do paciente. As variáveis adotadas para a identificação de ocorrência de lesão foram obtidas através do protocolo de Ottawa.

Na ocorrência de lesão, as nomenclaturas foram padronizadas, e, em seguida, a variável foi categorizada de maneira dicotômica (não ou sim) bem como as variáveis raio-x (não ou sim), e ocorrência de lesão (não ou sim), de acordo com cada examinador (acadêmico e residente).
Foram consideradas lesões as fraturas de maléolo medial, do maléolo lateral, fraturas bimaleolares, fraturas da base do $5^{\circ}$ metatarso, avulsões do ligamento deltoide, e avulsões do complexo ligamentar lateral evidenciáveis pelo exame radiográfico.

Para a descrição da amostra, foi realizada a distribuição de frequências (absoluta e relativa) da amostra total e segundo o examinador (acadêmico e residente). A capacidade do instrumento em identificar lesões de caráter verdadeiro positivo e verdadeiro negativo foi realizada utilizando análise de sensibilidade e especificidade, respectivamente, de acordo com a idade do paciente ( $\leq 54$ anos e $\geq 55$ anos). ${ }^{4} \mathrm{O}$ valor preditivo positivo indica em qual proporção os indivíduos podem ter um resultado do teste positivo e que realmente possuíam a característica/atributo, da mesma forma, o valor preditivo negativo busca predizer a real ausência da variável particularidade explorada. $^{5}$

Como medida de reprodutibilidade para concordância entre avaliadores (acadêmico e residente), foi feita através do teste do índice Kappa de Cohen pelos critérios propostos por Landis e Koch apud Barros et al. ${ }^{4}$ Para realizar as análises, foi utilizado o software SPSS 20.0 for Windows (IBM Corp., Armonk, NY, USA) e mantido um nível de significância em 5\% e determinados os cálculos de sensibilidade, especificidade, valor preditivo positivo (VPP) e VPN.

\section{Resultados}

Foram avaliados 263 pacientes ( 133 do sexo masculino e 130 do sexo feminino) com média de idades de 30,3 anos, sendo 22 pacientes com idade maior ou igual a 55 anos (8\%). Presença de fise aberta esteve evidente nas radiografias de 37 pacientes (13\%). A ocorrência de lesões foi determinada pela avaliação do médico plantonista e, posteriormente, pelo especialista somando um total de $23(8,7 \%)$ e 26 lesões $(9,9 \%)$, respectivamente (-Tabela $\mathbf{1})$.

Tabela 2 Análise das variáveis do protocolo de Ottawa e concordância entre acadêmico e residente na análise dos critérios* $(n=226)$

\begin{tabular}{|c|c|c|c|c|c|c|c|c|c|}
\hline \multirow[t]{2}{*}{ Variável } & \multirow[t]{2}{*}{ Categoria } & \multicolumn{2}{|c|}{ Acadêmico } & \multicolumn{2}{|c|}{ Residente } & \multirow[t]{2}{*}{ \% concordância } & \multirow[t]{2}{*}{ Kappa } & \multirow[t]{2}{*}{ Classificação $^{* *}$} & \multirow[t]{2}{*}{$p$} \\
\hline & & $\mathrm{N}$ & $\%$ & $\mathrm{~N}$ & $\%$ & & & & \\
\hline \multirow[t]{2}{*}{ Maléolo lateral } & Não & 86 & 38,1 & 118 & 52,2 & \multirow[t]{2}{*}{78,7} & \multirow[t]{2}{*}{0,580} & \multirow[t]{2}{*}{ Moderada } & \multirow[t]{2}{*}{$<0,001$} \\
\hline & Sim & 140 & 61,9 & 108 & 47,8 & & & & \\
\hline \multirow[t]{2}{*}{ Maléolo medial } & Não & 189 & 83,6 & 154 & 68,1 & \multirow[t]{2}{*}{80,1} & \multirow[t]{2}{*}{0,473} & \multirow[t]{2}{*}{ Moderada } & \multirow[t]{2}{*}{$<0,001$} \\
\hline & Sim & 37 & 16,4 & 72 & 31,9 & & & & \\
\hline \multirow[t]{2}{*}{ B5 } & Não & 202 & 89,4 & 170 & 75,2 & \multirow[t]{2}{*}{82,3} & \multirow[t]{2}{*}{0,413} & \multirow[t]{2}{*}{ Moderada } & \multirow[t]{2}{*}{$<0,001$} \\
\hline & Sim & 24 & 10,6 & 56 & 24,8 & & & & \\
\hline \multirow[t]{2}{*}{ Navicular } & Não & 196 & 86,7 & 169 & 74,8 & \multirow[t]{2}{*}{83,7} & \multirow[t]{2}{*}{0,471} & \multirow[t]{2}{*}{ Moderada } & \multirow[t]{2}{*}{$<0,001$} \\
\hline & Sim & 30 & 13,2 & 57 & 25,2 & & & & \\
\hline \multirow[t]{2}{*}{ Deambular } & Não & 174 & 77,0 & 145 & 64,2 & \multirow[t]{2}{*}{84,5} & \multirow[t]{2}{*}{0,643} & \multirow[t]{2}{*}{ Ótima } & \multirow[t]{2}{*}{$<0,001$} \\
\hline & Sim & 52 & 23,0 & 81 & 35,8 & & & & \\
\hline
\end{tabular}

*Protoco de Ottawa.

** por Landis e Koch. 
Tabela 3 Concordância entre ortopedista plantonista e especialista no raio x $(n=226)$

\begin{tabular}{|c|c|c|c|c|c|c|c|c|c|}
\hline \multirow[t]{2}{*}{ Variável } & \multirow[t]{2}{*}{ Categoria } & \multicolumn{2}{|c|}{$\begin{array}{l}\text { Ortopedista } \\
\text { Plantao }\end{array}$} & \multicolumn{2}{|c|}{$\begin{array}{l}\text { Ortopedista } \\
\text { Especialista }\end{array}$} & \multirow[t]{2}{*}{ \% concordância } & \multirow[t]{2}{*}{ Kappa } & \multirow[t]{2}{*}{ Classificação* } & \multirow[t]{2}{*}{$p$} \\
\hline & & $n$ & $\%$ & $\mathrm{~N}$ & $\%$ & & & & \\
\hline \multirow{2}{*}{$\begin{array}{l}\text { Ocorrência } \\
\text { de lesões }\end{array}$} & Não & 205 & 90,7 & 202 & 89,4 & \multirow[t]{2}{*}{98,7} & \multirow[t]{2}{*}{0,926} & \multirow[t]{2}{*}{ Perfeito } & \multirow[t]{2}{*}{$<0,001$} \\
\hline & Sim & 21 & 9,3 & 24 & 10,6 & & & & \\
\hline
\end{tabular}

*por Landis e Koch.

Obedecendo aos critérios de inclusão estabelecidos, restaram para a avaliação estatística 226 pacientes.

A aplicação das regras de Ottawa nos 226 pacientes pelos acadêmicos e residentes obteve os resultados mostrados na (-Tabela 2). A concordância obtida interobservador após a aplicação do método Kappa foi considerada moderada para as variáveis de palpação do maléolo medial, do maléolo lateral, e da base do $5^{\circ}$ metatarso e navicular, enquanto para variável incapacidade de deambular, o resultado da concordância foi ótimo $(0,643)$. Todos esses resultados tiveram significância estatística $(p<0,001)$. ( - Tabela 2)

$\mathrm{Na}$ avaliação radiográfica da ocorrência de lesões entre o ortopedista plantonista e o especialista em pé e tornozelo, foi evidenciada uma porcentagem de concordância de 98,7\% demostrando um índice Kappa considerado perfeito $(p<0,001)$ (-Tabela 3)

Foram analisados isoladamente os critérios do protocolo para acadêmicos e residentes e determinadas os valores de sensibilidade, especificidade, VPP e VPN. O teste mais sensível e com maior VPN para ambos, acadêmicos e residentes, foi a palpação do maléolo lateral, com sensibilidade de $83 \%$ e $78 \%$, respectivamente, e VPN de $96 \%$ para os dois grupos.

A análise estratificada dos dados do protocolo de Ottawa demonstrou uma sensibilidade para detectar fraturas de $100 \%$, e o VPN também foi $100 \%$ em nosso estudo. Do total de 203 pacientes, 61 tinham todos os critérios negativos, não necessitando de exame radiográfico e gerando uma redução de $30 \%$ no total de exames solicitados.

\section{Discussão}

Os traumas torcionais do tornozelo representam grande parte dos atendimentos nos serviços de urgência e emergência ortopédicos e sua avaliação deve buscar identificar as lesões ligamentares e fraturas que necessitem de tratamento imediato.

Sabe-se que do montante inicial de traumas da articulação tíbio-tarsica, somente 15\% incorrem em fraturas; logo, a dificuldade reside na diferenciação entre os pacientes que apresentam ou não lesão e quais deles demandam uma avaliação complementar.

$\mathrm{O}$ atendimento inicial desses pacientes cursa com uma avaliação radiográfica de pé e tornozelo de rotina, conduta essa que se mostra desnecessária na maioria das situações.

Tendo em vista os gastos sobressalentes ${ }^{6}$ com radiografias desnecessárias, o aumento do tempo desprendido no atendimento e a quantidade de radiação à qual o paciente é exposto, Stiell et al. ${ }^{3}$ criaram e validaram as regras do tornozelo de Ottawa, que, devido a sua praticidade e eficácia, tornaram-se uma ferramenta valiosa no atendimento ao trauma de tornozelo.

Bachmann et al. ${ }^{7}$ realizaram uma metanálise de 27 estudos incluindo cerca de 15.000 pacientes e encontraram dados que comprovam o protocolo como um instrumento clínico válido para a exclusão de fraturas do tornozelo e mediopé. As análises estatísticas demonstraram sensibilidade de 100\% e uma possibilidade de redução do número de radiografias solicitadas em 30 a $40 \%$ do total.

Rodrigues et al. ${ }^{8}$ validaram o Protocolo de Ottawa para a língua portuguesa em 2011 em Portugal, avaliando 123 pacientes demonstrando uma sensibilidade e VPN de $100 \%$ para detecção de fraturas com os critérios adotados pelo Protocolo e apresenta um potencial de redução do números de radiografias solicitadas no atendimento de emergência de cerca de 50\%. Em estudo realizado por pesquisadores brasileiros, Pires et al. ${ }^{9}$ concluiram que a incapacidade de suportar do peso na deambulação foi o item do protocolo com maior confiabilidade, apresentando $69,4 \%$ de sensibilidade, $61,6 \%$ especificidade, $63,1 \%$ de acurácia, $21,9 \%$ de valor preditivo positivo e $93 \%$ de valor preditivo negativo.

Stiell et al. ${ }^{10}$ e Ashurst et al. ${ }^{11}$ concluíram que a aplicação do Protocolo de Ottawa no atendimento de urgência/emergência acarretou em um menor tempo de atendimento destes pacientes em relação aos que foram submetidos à radiografia, $80 \mathrm{~min} / 116$ minute segundo Stiell. Ambos os estudos também concluem que não houve diferença significativa no grau de satisfação dos pacientes quando não submetidos a exames de imagens.

Contrapondo inúmeras publicações na literatura mundial sobre o tema, Kelly et al. ${ }^{12}$ apresentam resultados divergentes sobre a confiabilidade da avaliação pelo Protocolo de Ottawa, avaliando um total de 350 paciente em um estudo multicentrico foram encontradas 75 fraturas sendo que 5 não foram evidenciados pela regra de decisão clínica, sendo elas uma fratura instável do tornozelo, uma fratura do tálus, uma fratura do calcâneo e uma fratura do cuboide e do navicular. Evidenciando que as regras do protocolo devem ser associadas a um detalhado exame físico com atenção para condições de partes moles, como edema ou outros sinais clínicos sugestivos de fraturas, e a importância da deambulação presenciada pelo médico plantonista com a finalidade de afastar lesões graves da região tibio-talar e pé.

Em nossos resultados, encontramos um índice de concordância interobservador considerado moderado entre 
acadêmicos de medicina e residentes nos quesitos específicos de avaliação, entretanto essa diferença não alterou os valores de sensibilidade e VPN que permaneceram altos para ambos evidenciando um protocolo cuja taxa de falsos negativos foi nula.

$\mathrm{Na}$ avaliação entre o ortopedista plantonista e o especialista em pé e tornozelo, foi obtido um índice de concordância interobservador considerado perfeito, sendo que as possíveis lesões indicadas pelo protocolo foram diagnosticadas de maneira semelhantes, tanto na emergência quanto na avaliação pelo especialista.

Embora os bons resultados demonstrados no nosso estudo e comprovados na literatura indiquem a viabilidade da utilização das regras do tornozelo de Ottawa, encontramos algumas limitações na aplicabilidade clínica em nosso serviço, como a pressão dos pacientes para realização de exames radiográficos e a demanda por ganhos secundários.

A aplicação das regras do tornozelo de Ottawa para acadêmicos de medicina e residentes de ortopedia mostrou-se válida em nosso estudo, uma vez que os resultados obtidos corroboraram com os já presentes na literatura, sensibilidade e VPN de 100\% para ambos os grupos.

\section{Conclusões}

A aplicação do protocolo do tornozelo de Ottawa no atendimento inicial dos traumas torcionais de tornozelo dispensa com segurança a solicitação de exames radiográficos quando negativo para todos os critérios pesquisados, acarretando em um potencial de redução entre 30 e $50 \%$.

Os resultados mostraram uma aplicabilidade e concordância entre acadêmicos e residentes que permite a validação do protocolo do tornozelo de Ottawa para ambos os grupos nos atendimentos de urgência e emergência no Brasil.

Nossos dados estão de acordo com a literatura vigente, o que nos direciona a implementar o protocolo em nossa prática clínica, embora novos estudos sejam necessários para avaliar o impacto socioeconômico e cultural.
Conflito de Interesses

Os autores declaram não haver conflito de interesses.

\section{Referências}

1 Wang X, Chang SM, Yu GR, Rao ZT. Clinical value of the Ottawa ankle rules for diagnosis of fractures in acute ankle injuries. PLoS One 2013;8(04):e63228

2 Waterman BR, Belmont PJJr, Cameron KL, Deberardino TM, Owens BD. Epidemiology of ankle sprain at the United States Military Academy. Am J Sports Med 2010;38(04):797-803

3 Stiell IG, Greenberg GH, McKnight RD, Nair RC, McDowell I, Worthington JR. A study to develop clinical decision rules for the use of radiography in acute ankle injuries. Ann Emerg Med 1992;21(04):384-390

4 Barros MV, Reis RS, Hallal PC. Análise de Dados em Sáude. 3rd ed. Londrina: Midiograf; 2012

5 Jekel JF, Elmore JG, Katz DL. Introdução à medicina preventiva. In: Jekel JF, Elmore JG, Katz DL, editores. Epidemiologia bioestatística e medicina preventiva. Porto Alegre: Artmed; 1999: 203-209

6 Anis AH, Stiell IG, Stewart DG, Laupacis A. Cost-effectiveness analysis of the Ottawa Ankle Rules. Ann Emerg Med 1995;26 (04):422-428

7 Bachmann LM, Kolb E, Koller MT, Steurer J, ter Riet G. Accuracy of Ottawa ankle rules to exclude fractures of the ankle and mid-foot: systematic review. BMJ 2003;326(7386):417

8 Rodrigues P, Rosa I, Campagnolo JL. [Validation of the Ottawa rules for the Portuguese population: a prospective study]. Acta Med Port 2011;24(05):713-718

9 Pires R, Pereira A, Abreu-E-Silva G, et al. Ottawa ankle rules and subjective surgeon perception to evaluate radiograph necessity following foot and ankle sprain. Ann Med Health Sci Res 2014;4 (03):432-435

10 Stiell IG, McKnight RD, Greenberg GH, et al. Implementation of the Ottawa ankle rules. JAMA 1994;271(11):827-832

11 Ashurst JV, Nappe T, Digiambattista S, et al. Effect of triage-based use of the Ottawa foot and ankle rules on the number of orders for radiographic imaging. J Am Osteopath Assoc 2014;114(12): 890-897

12 Kelly AM, Richards D, Kerr L, et al. Failed validation of a clinical decision rule for the use of radiography in acute ankle injury. $\mathrm{NZ}$ Med J 1994;107(982):294-295 